\title{
Cutting-Edge Reproductive Medicine and Ethics in Japan
}

\author{
Masayuki Kodama \\ National Institute of Fitness and Sports in Kanoya
}

\begin{abstract}
Although the Japan Society of Obstetrics and Gynecology (JSOG) endorses new-type PGD using CGH for only carriers of balanced chromosomal translocations, it is against the ethical guidelines of the JSOG to decide whether or not embryos should be implanted due to aneuploidy discovered as a result of new-type PGD. In the author's opinion, it should be at the discretion of the JSOG's own randomized controlled trials, which should involve multiple facilities, whether or not a scientific basis can be found for the value of the new-type PGD in cases of recurrent miscarriage, in cases of implantation failure, and in cases where the women are of advanced maternal age. Dr. Netsu's 36 cases of selective reduction may be against the Japanese Maternal Health Protection Law that prohibits abortion due to congenital disease, but with the backdrop that there are many abortions that are performed as a result of parental convenience in Japan, it is difficult to understand the difference between the over 300,000 abortions that are performed every year in Japan and Dr. Netsu's prescribed surgery which is designed to selectively reduce fetuses with congenital disease for the purpose of evading physical danger for women with multiple gestations.
\end{abstract}

Keywords: new type preimplantation genetic diagnosis (new-type PGD), Japan Society of Fertilization and Implantation (JSFI), Japan Society of Obstetrics and Gynecology (JSOG), selective reduction, Yahiro Netsu

\section{Historical Trends in “Cutting-Edge Reproductive Medicine and Ethics in Japan”}

Cutting-edge reproductive medicine is not legally regulated in Japan. The Assisted Reproductive Technologies (ART) Review Committee of the Science Council of Japan (December 21st, 2006 to April 30th, 2008; ART Review Committee) summarized their conclusions after deliberation lasting more than one year on April 8th, 2008. However, despite the report of these conclusions entitled "Issues Related to Assisted Reproductive Technologies Centered on Surrogate Pregnancy—-Toward a Social Consensus” to the Ministry of Education, Culture, Sports, Science, and Technology (MEXT) and Ministry of Justice (MOJ) in Japan, cutting-edge ART has still not been discussed by the national diet in Japan as of January 2014. During the six years since the Japanese government received the ART Review Committee report, this failure to address the issue of patients who receive ART treatment has become a serious issue in society at large.

Amidst these unusual circumstances, Japanese patients who cannot wait until the end of their reproductive age are increasingly traveling abroad to receive cutting-edge ART treatment. However, instead of going to the United States, where the expenses, including treatment and travel costs, are high, Japanese patients are now increasingly traveling to countries like India and Thailand that provide high-quality ART treatment at lower

Masayuki Kodama, Ph.D., doctor of philosophy, Research on Preimplantation Genetic Diagnosis in Japan, Faculty of Physical Education, National Institute of Fitness and Sports in Kanoya (NIFS), Japan; main research field: Reproductive Medical Ethics. Email: kodama@nifs-k.ac.jp. 
costs. Since the Manji Yamada incident ${ }^{1}$ in July 2008, it is ironic that surrogate births from India, with low-cost yet high-quality care similar to the United States, are now increasingly common in Japan. Thus, the number of Japanese patients traveling to India and Thailand for surrogate pregnancies is rapidly increasing.

As of February 2011, among Japanese couples seeking surrogate births using overseas clinics, at least 20 went to India and 10 went to Thailand. Among an estimated 250 or more in Vitro Fertilization and Embryo Transfer (IVF-ET) clinics in India, the Akanksha Infertility Clinic, where Dr. Nayna Patel is the medical director, was featured in an on-site report ${ }^{2}$ by an Asahi Shimbun journalist in 2011. There had been eight Japanese surrogate births and one that was a current pregnancy. Therefore, as of January 2014, the number of Japanese surrogate births is certainly in the double digits.

According to Dr. Somboon Kunathicom who worked in a hospital in Bangkok, in Thailand, among 908 cases of Preimplantation Genetic Diagnosis (PGD) in 2010, “80\% involved gender selection.” In addition, the number of Japanese couples going to Thailand for gender selection using PGD has been increasing yearly, from 50 in 2009 to 61 in 2010, and to 103 in 2011. Gender selection using the new-type PGD, a technique in which all chromosomes are analyzed, began in February 2012. The number of Japanese couples who opted for gender selection using new-type PGD reached 90 by July 2012, and one Bangkok intermediary estimated that this number would exceed 200 couples by the end of 2012. ${ }^{3}$

In Japan, at the Otani Ladies Clinic, where Dr. Tetsuo Otani was the medical director, new-type PGD was used to test for chromosome numerical abnormalities with the goal of preventing miscarriages (with a total of 97 patients between February 2011 and May 2012). As a result of using new-type PGD in these 97 women who had frequent miscarriages (mean age: 39.1), among the 53 women with uterine implantation of a fertilized egg, 39 became pregnant and 16 gave birth. These results by Dr. Otani were announced in the Yomiuri Shimbun on July 11th, 2012.

Meanwhile, Dr. Yahiro Netsu, who is the medical director of Suwa Maternity Clinic, and continues to raise the issue of ART for patient relief to the government and society, opposes the prohibition of surrogate pregnancy by the Japan Society of Obstetrics and Gynecology (JSOG; April 2003). Dr. Netsu has helped 21 patients with surrogate births as of January 2014.

\section{Conditional Approval of CGH and Selective Reduction for Congenital Abnormalities}

Given this background of historical trends on "cutting-edge reproductive medicine and ethics in Japan," the Japan Society of Fertilization and Implantation (JSFI) held its 31st Annual Meeting in Oita on August 8th-9th, 2013. This meeting included an invitational lecture by Dr. Santiago Munné from the United States entitled "PGD and carrier screening for infertile couples," and a symposium on the "Future of PGD" (Chairperson: Dr. Kou Sueoka). At this symposium, comparative genomic hybridization (CGH), which enabled comprehensive analysis of the entire genome and was an essential technique for new-type PGD, was conditionally approved. In other words, the application of new-type PGD using CGH was approved with limitation to chromosomal translocation carriers. In summary, many of the symposiasts express a view that application of preimplantation genetic screening (PGS) that is limited to chromosomal translocation carriers is realistic. When one considers the possibility that PGS is a result of innovative changes in diagnostic technology, a majority of the symposiasts also express an opinion that allowing application of PGD for chromosomal numerical abnormalities, in other words, conditional approval of PGS using CGH technology (new-type PGS), would also be realistic. 
At this JSFI meeting, Dr. Yahiro Netsu announced his "Study of 1,001 Cases of Selective Reduction." Since 1986 when Dr. Netsu first began performing multi-fetal pregnancy reduction/selective reduction in Japan, selective reduction had been performed in 1,001 patients as of February 2013 (including 921 cases with consultation after a multiple pregnancy was found at other facilities). Moreover, in order to prevent health risks to pregnant women with multiple fetuses, selective reduction was performed for fetuses with congenital abnormalities in 36 cases.

\section{Request by JSFI Symposiasts and the Issue of Dr. Netsu's Violation of the Law}

The approval of PGD using CGH at the JSFI symposium with limitation to chromosome translocation carriers (preceding Section 2, first paragraph) is an issue that may force reconsideration of the clinical application of new-type PGD in cases of chromosomal numerical abnormalities (aneuploidy), in other words, reconsideration of the JSOG ethical guidelines, which have not yet approved new-type PGS.

In addition, Dr. Otani and colleagues, who applied the use of new-type PGD, without request to the JSOG, for screening of chromosomal numerical abnormalities (97 patients between February 2011 and May 2012), published their clinical results on July 11th, 2012 (74\% pregnancy rate in women with a mean age of 39 who had uterine implantation). ${ }^{4}$ Despite these groundbreaking (especially when one considered the $25 \%$ pregnancy rate with standard IVF-ET) results, the JSOG chairman-Professor Ikuo Konishi of Kyoto University, maintained a firm stance against the clinical application of new-type PGD (namely, new-type pre-implantation genetic screening, PGS) for screening of chromosomal numerical abnormalities (aneuploidy). On July 27th, 2012, the JSOG released the "Declaration of the JSOG Regarding Media Coverage of PGD.” In other words, the JSOG still has not altered their critical stance ${ }^{5}$ regarding new-type PGS.

Meanwhile, regarding selective reduction for fetal congenital abnormalities by Dr. Netsu (preceding Section 2, last paragraph), it is unclear whether induced abortions, which are allowed under certain circumstances by the Maternal Health Protection Law (a revision in 1996 from the Eugenic Protection Law enacted in 1948), by definition also include selective fetal reductions. Nevertheless, it cannot be denied that this may violate the Japanese Maternal Health Protection Law, which prohibits induced abortions for congenital abnormalities.

\section{The Purpose of This Paper}

This paper attempts to add to the discussion regarding two issues concerning "cutting-edge reproductive medicine and ethics in Japan" as discussed in the preceding Section 3.

First, the paper will discuss a "request by JSFI symposiasts to reconsider the JSOG ethical guidelines." Specifically, this is a proposal to the JSOG concerning a way to resolve the "discrepancy between CGH conditional approval by JSFI symposiasts and the JSOG ethical guidelines.”

Next, this paper will discuss the "issue of Dr. Netsu's violation of the Maternal Health Protection Law." Specifically, this is an "ethical discussion regarding selective induced abortions of fetuses with congenital abnormalities in multiple pregnancies.”

\subsection{Resolving the Discrepancy between Conditional Approval of CGH and the JSOG Ethical Guidelines}

Significance of Conditional Approval of CGH. The conditional approval of CGH technology for use limited to chromosome translocation carriers has significance in and of itself. In fact, even if a fertilized egg 
without any chromosome translocations is selected from a group of fertilized eggs of a known chromosome translocation carrier, there is still a high (20\%) miscarriage rate after PGD of a chromosome translocation. These high miscarriage rates include cases where aneuploidy (a chromosomal numerical abnormality) is the cause. If PGS using CGH is performed to exclude fertilized eggs with aneuploidy, the miscarriage rate after PGD in known chromosome translocation carriers can be reduced to $7-10 \%{ }^{6}$. Therefore, the conditional approval of CGH technology for use limited to chromosome translocation carriers is the first step toward lifting the ban on PGS (new-type PGS) using CGH.

Voices of Doubt about Conditional Approval of CGH. Even with conditional approval of CGH expressed at the JSFI symposium, some experts are saying that if the application of CGH is limited to chromosome translocation carriers, the purpose for the lifting of the prohibition of CGH comes under question.

Of course, if bioethical issues regarding cutting-edge reproductive medicine (life selection and discrimination against the disabled) are taken into account, then, for detection of chromosome translocations in a fertilized egg from a chromosome translocation carrier, fluorescence in situ hybridization (FISH) may be preferable for obtaining information that is focused on the site of a target chromosomal structural abnormality. However, if we follow gynecologic evidence for preventing miscarriages due to chromosomal abnormalities (structural abnormalities and numerical abnormalities), ${ }^{7}$ then $\mathrm{CGH}$ is a revolutionary technique that can discover all types of chromosomal abnormalities, some of which cannot be detected by FISH, which is limited to analyzing genetic information on the chromosome. This is best exhibited precisely by using CGH for analysis of genomic abnormalities, including chromosome translocations.

Therefore, it is concluded that the JSFI should promptly and comprehensively re-examine their conditional approval of CGH. This includes analysis: (i) from a medical perspective in order to evaluate whether PGS using CGH (new-type PGS, namely, new-type PGD for aneuploidy testing) is effective; (ii) from an ethical perspective in order to examine criticisms concerning life selection and discrimination towards the disabled; and (iii) from a realistic perspective in order to provide timely help to infertile patients who are still of reproductive age and want to prevent a miscarriage. This is the sincere intent behind the voices of doubt regarding conditional approval of $\mathrm{CGH}$.

However, the bioethics of reproductive medicine is a difficult issue that concerns both the JSFI and JSOG. The urgent issue for the JSFI and JSOG, based on the assumption that the justification for their existence as medical societies is to clearly present medical evidence, is therefore to objectively demonstrate whether PGS using CGH (new-type PGS) is effective.

Proposal for the JSOG Itself to Conduct Multicenter Randomized Controlled Trials. Implementation of PGS using CGH technology currently violates the JSOG ethical guidelines (Declaration of the JSOG Regarding Media Coverage of PGD; July 27th, 2012).

Point 2 of the JSOG "Declaration" is the "concern that this media coverage (the announcement by Dr. Tetsuo Otani about the revolutionary clinical results of PGS using CGH technology) would inevitably lead to inappropriate widespread information to the general public.” The reasons behind this statement include insufficient scientific evidence for the assertion that new-type PGS, namely, new-type PGD for aneuploidy testing, will "increase pregnancy rates" and is "good news for infertility" (as reported in the Yomiuri Shimbun article dated July 11th, 2012). They believe that this "might lead to unrealistic expectations and misunderstandings by the public." 
"A recent meta-analysis (an analysis of many previously published reports) found that PGS (author's note: new-type PGS) did not contribute to improved pregnancy rates or live birth rates." "The European Society of Human Reproduction and Embryology (ESHRE: 2010) also failed to find any scientific evidence showing the efficacy of PGS (author's note: new-type PGS) in recurrent miscarriages, implantation failure, or older women. Therefore, they stated that there was a need to conduct multicenter collaborative Randomized Controlled Trials (RCTs)."

Following the author's expressed doubts about the JSOG ethical guidelines in a paper ${ }^{8}$ entitled "An Ethical View on the Initial Clinical Application of the New Type Preimplantation Genetic Diagnosis of Embryos in Japan” in September 2013, the American Society for Reproductive Medicine (ASRM), as it happened, also published during the same month articles ${ }^{9}$ in their journal (Fertil Steril, September 2013) as the results of a multicenter RCT supporting the efficacy of new-type PGS, namely, new-type PGD for aneuploidy testing.

Despite the American College of Obstetricians and Gynecologists (ACOG) Committee Opinion No. 430 published in March 2009, and the ESHRE announcement in 2010, in which the JSOG bases its reason for not altering their stance on the clinical application of new-type PGS (PGS using CGH, Single Nucleotide Polymorphism—SNP arrays, and quantitative PCR), all of the clinical data that was used was based on old-type PGS (PGS using FISH). The assertion of this as scientific evidence to negate the scientific efficacy of new-type PGS (PGS using CGH) is a misguided mistake, as it has already been described. ${ }^{10}$

With regard to whether the JSOG ethical guidelines are correct and will stand the test of time, namely, strictly regulating new-type PGS (new-type PGD for aneuploidy testing), the JSOG should follow the lead of the $\mathrm{ASRM}^{11}$ and conduct their own multicenter RCTs in order to draw their own objective and non-biased conclusions. Without the JSOG conducting their own multicenter collaborative RCTs, they cannot scientifically verify if and/or when new-type PGS is effective.

\subsection{Ethical Considerations on Dr. Netsu's Selective Reduction}

Selective Induced Abortions in Multiple Pregnancies. Even before his presentation at the JSFI (August 8th, 2013), Dr. Netsu told the Yomiuri Shimbun (August 5th, 2013) that he had performed 36 selective reductions for fetal abnormalities in women with multiple pregnancies. The reason for selective reduction was a chromosomal abnormality such as Down's syndrome in 25 cases and fetal hydrops in 11 cases.

Under the current Maternal Health Protection Law, performing an abortion for a fetal congenital abnormality is illegal. If congenital abnormalities are found in fetuses, a pregnant woman is forced to decide to try and give birth to all of the fetuses, or to have an abortion of all of the fetuses.

Therefore, the explanation given by Dr. Netsu regarding the current law to the couples in these 36 cases of selective reduction meant that without exception, "unless selective reduction is performed, all the fetuses will be aborted.” Thus, "to save at least one life, this is something that needs to be done.” Among these 36 cases of selective reduction, 32 women gave birth; one had a miscarriage; and three were currently pregnant or had an unknown pregnancy status. ${ }^{12}$ Among the 36 women, 31 had multiple pregnancies after infertility treatment (ovulation-inducing drugs or multiple embryo transfers). These selective abortions were performed at 12 weeks of pregnancy or later, when a stillbirth notification is required. ${ }^{13}$

The Ministry of Health, Labour, and Welfare (MHLW) Council issued a report in 2000 stating that selective reduction "should, in principle, not be performed," but in cases of triplets or higher order pregnancies, 
an exception can be made if selective reduction is necessary. However, even in such cases, selective reduction is not approved for fetal abnormalities or gender selection.

Dr. Netsu, in the Yomiuri Shimbun article (August 5th, 2013) gave the following reason for performing selective reduction, even though he was aware that this statement and procedure violated the Maternal Health Protection Law: "The number of married couples who want selective reduction because of a fetal abnormality is going to increase in the future. Society as a whole should consider the significance of fetal reduction in cases where at least one fetus survives the procedure.”

Ethical Considerations. Amidst these background ethical issues being raised in society about Dr. Netsu's selective reduction, prenatal diagnosis (PND) in pregnant women has improved in the last few years. For example, in prenatal examinations, ultrasonography is usually performed before amniocentesis for a confirmatory diagnosis. Ultrasound technology has rapidly expanded the ability to assess healthy fetal development. Ultrasound can now be used to study fetal growth and sex determination, as well as to assess chromosomal abnormalities and cardiac disease. Since April 2013, new-type PND, namely, non-invasive prenatal genetic testing (NIPT), in which congenital diseases such as Down's syndrome (trisomy 21), Edwards syndrome (trisomy 18), and Patau syndrome (trisomy 13) can be detected simply by blood tests, has also been approved by the JSOG.

The JSOG, together with the Japan Medical Association, Japanese Association of Medical Sciences, Japan Association of Obstetricians and Gynecologists, and Japan Society of Human Genetics, issued a joint statement on March 9th, 2013 entitled "New Prenatal Genetic Testing Using Maternal Blood.” This statement declares that NIPT should be performed only in facilities that can provide full genetic counseling in the following types of patients: (1) older pregnant woman; (2) women in whom ultrasonography or maternal serum markers suggest that the fetus has a chromosomal numerical abnormality; (3) women who have already been pregnant with a child who has a chromosomal numerical abnormality; or (4) when either parent has a balanced Robertsonian translocation and the fetus may have trisomy 13 or trisomy 21.

As a result, the ban on NIPT was lifted in April 2013, and among 7,775 pregnant women who had NIPT during the one-year period that ended in March 2014, "148 women (1.8\%) had a high likelihood (positive) of having a fetus with disease.” Moreover, since NIPT was introduced, "More than $90 \%$ of the pregnant women in whom fetal disease was diagnosed by six months elected to have pregnancy termination."

Within the background of this social situation, the current Japanese Maternal Health Protection Law does not permit abortions because of a fetal abnormality. However, in actual clinical practice, due to loopholes in the Japanese Maternal Health Protection Law, abortions (more than 1,000 each year ${ }^{15}$ ) are being performed for "medical reasons (health risks)," "social reasons (economical)," and "ethical reasons (rape)."

Abortions based on the results of PND where fetal diagnosis or testing after pregnancy is established which includes chorionic villus sampling, amniocentesis, fetoscopy, fetal umbilical blood tests, and ultrasound, are being tacitly allowed by society under the guise of the Japanese Maternal Health Protection Law (e.g., for economic reasons). Among these abortions being performed for various social reasons, there are presumably many that could have been and should be avoided. Once considering the fact that many abortions are actually being performed in clinical practice, it is difficult to find any clear ethical or moral differences between these abortions and the selective reductions being performed by Dr. Netsu for fetal congenital abnormalities in multiple pregnancies. 


\section{Notes}

1. The incident where a girl (born on July 25th, 2008) born to an Indian surrogate mother impregnated by IVF-ET of a fertilized embryo inseminated by Dr. Nayna Patel using an egg cell received by a Japanese physician from an anonymous Nepalese donor temporarily became stateless.

2. Asahi Shimbun (Tokyo morning edition), February 19th, 2011.

3. Yomiuri Shimbun (Tokyo morning edition), July 16th, 2012.

4. According to the Yomiuri Shimbun (Tokyo morning edition), July 11th, 2012, Dr. Tetsuo Otani had on his own initiative, without approval from the JSOG, implemented new style PGD in numerical chromosomal abnormality tests for the prevention of abortion (97 cases from February 2011 to May 2012). Dr. Otani's PGD treatment results were revolutionary (e.g., of the 97 frequent miscarriage patients with an average age of 39.1, 53 patients had successful fertilized egg cell womb implantation, of whom 39 became pregnant and 16 gave birth. In comparison with the $25 \%$ pregnancy rate with the common IVF-ET transplantation procedure, the $74 \%$ pregnancy rate among successful fertilized egg cell womb implantation patients with an average age of 39 is 3 times as high).

However, his violation of JSOG regulations (guiding principles) was without argument. JSOG chairman and Kyoto University professor, Ikuo Konishi, who dealt with the media coverage over this violation, published in the same newspaper on the same day the following opinion on the augmentation of PGD implementation: "There are members of national disability groups who are opposed to the technology, so we must widely and purposely debate the problem of implementation of new style PGD within our society in order to gain consensus. We cannot allow technology to take the lead.”

5. On July 27th, 2012, the JSOG clearly showed its stance with the release of the Declaration of the Japan Society of Obstetrics and Gynecology Regarding Media Coverage Concerning "Preimplantation Genetic Diagnosis" under the signatures of JSOG chairman Ikuo Konishi, and Ethical Committee chairman Kazunori Ochiai. Two points were made in this release.

With regard to cases of unauthorized performance (of, author's note: PGS) other than those such cases deemed suitable by this Society, we clearly state that this conduct will never be approved.

We express our concerns that through media coverage inappropriate information concerning this problem has been widely dispersed within society.

6. David R. Meldrum. Preimplantation Genetic Screening is Alive and Very Well. Fertil Steril, 2013, 100 (3): 593-4.

Hodes-Wertz et al.. Idiopathic Recurrent Miscarriage is Caused Mostly by Aneuploidy Embryos. Fertil Steril, 98 (3): 675-80.

Grifo, S. Ghadir et al. (ASRM 2011). Significant Decrease in Miscarriages After Preimplantation Genetic Diagnosis (PGD) for Recurrent Pregnancy Loss Using Array Comparative Genome Hybridization (array CGH). (O-76 Monday, October 17th, 2011 05:45 p.m.)

7. "Chromosomal abnormality" is divided into "structural abnormality" and "numeral abnormality." "Structural abnormality" includes not only "chromosomal translocation" which is subdivided into "reciprocal translocation" and "Robertsonian translocation," but also "inversion," "deletion," and "duplication,” while "numeral abnormality” is subdivided into "aneuploidy" and "polyploidy."

8. Masayuki Kodama. An Ethical View on the Initial Clinical Application of the New Type Preimplantation Genetic Diagnosis of Embryos in Japan. Bioethics (Seimei Rinri), 2013, 24: 63-71.

9. Treff et al.. Blastocyst Biopsy with Comprehensive Chromosome Screening and Fresh Embryo Transfer Significantly Increases in Vitro Fertilization Implantation and Delivery Rates: A Randomized Controlled Trial. Fertil Steril, 2013,100 (3): 697-703.

David R. Meldrum. Preimplantation Genetic Screening is Alive and Very Well. Fertil Steril, 2013, 100 (3): 593-4.

Katherine L. Scott et al.. Selecting the Optimal Time to Perform Biopsy for Preimplantation Genetic Testing. Fertil Steril, 2013, 100 (3): 608-14.

William B. Schoolcraft et al.. Comprehensive Chromosome Screening of Trophectoderm with Vitrification Facilitates Elective Single-embryo Transfer for Infertile Women with Advanced Maternal Age. Fertil Steril, 2013, 100 (3): 615-9.

N. R.Treff et al. (ASRM 2013). Development and Validation of a Next-generation Sequencing (NGS)-based 24-chromosome Aneuploidy Screening System. (O-270 Tuesday, October 15th, 2013 04: 15 p.m.)

Alan H. Handyside. 24-chromosome Copy Number Analysis: A Comparison of Available Technologies. Fertil Steril, 2013, 100 (3): 595-602.

Nathan R. Treff et al.. Cleavage-stage Biopsy Significantly Impairs Human Embryonic Implantation Potential while Blastocyst Biopsy does not: A Randomized and Paired Clinical Trial. Fertil Steril, 2013, 100 (3): 624-30.

10. Masayuki Kodama. Ibid..

11. Research results proving the scientific efficacy of new-type PGS (PGS using CGH) were offered in presentations and published papers at the 69th ASRM annual meeting held on October 10th-12th, 2013 in conjunction with IFFS at the Boston Convention \& Exhibition Center, Boston, Massachusetts, USA, and in the ASRM's journal (Fertil Steril, September 2013).

12. Yomiuri Shimbun (Tokyo morning edition), August 5th, 2013.

13. Yomiuri Shimbun (Tokyo morning edition), August 6th, 2013.

14. Yomiuri Shimbun (Tokyo morning edition), April 20th, 2014.

15. Yomiuri Shimbun (Tokyo morning edition), August 6th, 2013. 


\section{Works Cited}

Keltz M.D., et al.. "Preimplantation Genetic Screening (PGS) with Comparative Genomic Hybridization (CGH) following Day 3 Single Cell Blastomere Biopsy Markedly Improves IVF Outcomes while Lowering Multiple Pregnancies and Miscarriages.” J Assist Reprod Genet, 30.10 (2013): 1333-9.

Santiago Munné, et al.. "Preimplantation Genetic Diagnosis for Aneuploidy and Translocations Using Array Comparative Genomic Hybridization.” Current Genomics, 13.6 (2012): 463-70.

Adler A., et al.. "Blastocyst Culture Selects for Euploid Embryos: Comparison of Blastomere and Trophectoderm Biopsies." Reprod Biomed Online, 28.4 (2014): 485-91.

Santiago Munné, et al.. "Diminished Effect of Maternal Age on Implantation after Preimplantation Genetic Diagnosis with Array Comparative Genomic Hybridization.” Fertil Steril, 100.6 (2013): 1695-703.

Ermanno Greco, et al.. "Comparative Genomic Hybridization Selection of Blastocysts for Repeated Implantation Failure Treatment: A Pilot Study.” BioMed Research International. Vol. 2014, 10.

ESHRE PGD Consortium data collection XII: Cycles from January to December 2009 with Pregnancy Follow-up to October 2010. Hum Reprod, 29 (2014): 880-903.

Santiago Munné, et al.. “Biomarkers for Infertility and Recurrent Pregnancy Loss.” Reprod Biomed Online. 2014, in press.

Santiago Munné, et al.. "Genome Wide Karyomapping Accurately Identifies the Inheritance of Single Gene Defects in Human Preimplantation Embryos in Vitro.” Genetics in Medicine. 2014, in press.

Santiago Munné, et al.. “A Greater Number of Euploid Blastocysts in a Given Cohort Predicts Excellent Outcomes in Single Embryo Transfer Cycles.” JARG. 2014, in press.

Grifo J.A., et al.. "Live Birth from Previously Vitrified Oocytes, After Trophectoderm Biopsy, Revitrification, and Transfer of a Euploid Blastocyst.” Clin Med Insights Reprod Health, (7) 2013: 79-82.

Chavez S.L., et al.. "Dynamic Blastomere Behaviour Reflects Human Embryo Ploidy by the Four-cell Stage.” Nature Communications, 3 (2012):1251.

Kroener L., et al.. “The Effect of Timing of Embryonic Progression on Chromosomal Abnormality.” Fertil Steril, 98.4 (2012): 876-80.

Colls P., et al.. "Validation of Array Comparative Genome Hybridization for Diagnosis of Translocations in Preimplantation Human Embryos.” Reprod Biomed Online, 24 (2012): 621-9.

Harton G., et al.. "Microarrays and CGH for PGD of Chromosome Abnormalities and Gene Defects. In: Practical Manual of in Vitro Fertilization: Advanced Methods and Novel Devices.” Nagy et al.. 2012, Chapter 53, 483-90.

Ata B., et al.. "Array CGH Analysis Shows that Aneuploidy is not Related with the Number of Embryos Generated." Reprod Biomed Online, 24 (2012): 614-20.

Santiago Munné, et al.. “Intra-age, Intercenter, and Intercycle Differences in Chromosome Abnormalities in Oocytes.” Fertil Steril, 97 (2012): 935-42.

Bisignano A., et al.. "Preimplantation Genetic Diagnosis and Aneuploidy Screening for 24 Chromosomes: Advantages and Disadvantages of Competing Platforms.” Reproductive Biomed Online, 23.6 (2011): 677-85.

Santiago Munné, et al.. Culture-induced Chromosome Abnormalities: The Canary in the Mine. Reproductive Biomed Online, 2011, 22: $506-8$.

Gutiérrez-Mateo C., et al.. "Validation of Microarray Comparative Genomic Hybridization for Comprehensive Chromosome Analysis of Embryos.” Fertil Steril, 95 (2011): 953-8.

Magli M.C., et al.. “Aneuploidies of Chromosomes 1, 4 and 6 are not Compatible with Human Embryos’ Implantation.” Fertil Steril, 2010. 2012-6.

Santiago Munné, et al.. "Microarrays and CGH for PGD of Chromosome Abnormalities and Gene Defects.” Journal of Reproductive Medicine and Endocrinology, 7 (2010): 236-9.

Schoolcraft W.B., et al.. "Clinical Application of Comprehensive Chromosomal Screening at the Blastocyst Stage.” Fertil Steril, 94 (2010): 1700-6.

Fragouli E., et al.. "Comprehensive Chromosome Screening of Polar Bodies and Blastocysts from Couples Experiencing Repeated Implantation Failure.” Fertil Steril, 94 (2010): 875-87. 
Fischer J., et al.. "Preimplantation Genetic Diagnosis (PGD) Improves Pregnancy Outcome for Translocation Carriers with a History of Recurrent Losses.” Fertil Steril, 94 (2010): 283-9.

Santiago Munné, et al.. “Technology Requirements for Preimplantation Genetic Diagnosis to Improve Art Outcome.” Fertil Steril, 94 (2010): 408-30.

Santiago Munné. “Overview of Preimplantation Genetic Diagnosis.” Expert Rev. Obstet Gynecol, 5 (2010): 403-8.

Santiago Munné, et al.. “An Improved 12-Chromosome FISH Test Could Detect 91\% of Aneuploid Blastocysts.” Reprod Biomed Online, 20 (2010): 92-97. 